\title{
Análisis de coordenadas polares para el estudio de los sistemas defensivos en balonmano
}

\section{Polar coordinate analysis for the study of defensive systems in Handball}

\section{Análise de coordenadas polares para o estudo de sistemas defensivos no handebol.}

\author{
Jiménez-Salas, J. ${ }^{1}$, Morillo-Baro, J.P. ${ }^{2}$, Reigal, R.E. ${ }^{3}$, Morales-Sánchez, V. ${ }^{4}$, Hernández-Mendo, A. ${ }^{5}$ \\ Facultad de Psicología, Universidad de Málaga ${ }^{1,2,3,4,5}$
}

\section{RESUMEN}

El objetivo de este estudio fue analizar la relación de diferentes comportamientos tácticos ofensivos con las conductas mostradas en los dos principales sistemas defensivos en balonmano. Para ello se realizó un análisis de coordenadas polares utilizando como conductas focales aquellas que identifican los principales sistemas defensivos y las acciones ofensivas con o sin transformaciones. Se observaron 538 situaciones correspondientes a 5 partidos de la primera división nacional española masculina con el software Hoisan utilizándose una herramienta observacional diseñada ad hoc. El diseño observacional usado fue nomotético, puntual y seguimiento, siendo la unidad de observación la situación de defensa del equipo observado. Los resultados del análisis de la Calidad del Dato y Generalizabilidad muestran índices convincentes para los modelos utilizados. Los resultados del análisis de Coordenadas Polares muestran la mayor capacidad de oposición del sistema defensivo 5:1 al ataque. También destaca que la transformación atacante de 3:3 a 2:4 se usa más ante defensas abiertas y elicita el aumento de aparición de sanciones disciplinarias. La herramienta observacional diseñada y la utilización de la técnica de coordenadas polares para la estimación de relaciones tácticas permite definir procedimientos de intervención que optimicen los recursos del entrenamiento y la competición en balonmano.

Palabras clave: Observación Sistemática, Coordenadas Polares, Balonmano, Defensa.

\begin{abstract}
The objective of this study was to analyze the relationship of different offensive tactical performances with the behaviours shown in the two main defensive systems in handball. For this, an analysis of polar coordinates was carried out using as focal behaviors those wich identify the main defensive systems and the offensive actions with or without transformations. There were 538 situations corresponding to 5 matches of the first Spanish national men's division with the Hoisan software by making use of an ad hoc tool. The observational design used was nomothetic, punctual and monitoring, the observation unit being the defense situation of the monitored team. The results of the Data Quality and Generalizability analysis show convincing rates for the models used. The results of the Polar Coordinates analysis show the greater oppositional capacity of the 5: 1 defensive system to attack. It also highlights that the attacking transformation from 3:3 to 2:4 is used more in the face of open defenses and it elicits an increase in the appearances of disciplinary sanctions. The observational tool designed and the use of the polar coordinate
\end{abstract}




\section{Jiménez-Salas, Morillo-Baro, Reigal, Morales-Sánchez, Hernández-Mendo}

technique for the estimation of tactical relationships allows to define intervention procedures that optimize the resources of training and competition in handball.

Keywords: Systematic Observation, Polar Coordinates, Handball, Defense.

\section{RESUMO}

O objetivo deste estudo foi analisar a relação de diferentes comportamentos táticos ofensivos com os comportamentos mostrados nos dois principais sistemas defensivos do handebol. Para tanto, foi realizada uma análise das coordenadas polares utilizando como comportamentos focais aqueles que identificam os principais sistemas defensivos e as ações ofensivas com ou sem transformações. Havia 538 situações correspondentes a 5 partidas da primeira divisão masculina nacional espanhola com o software Hoisan, usando uma ferramenta projetada ad hoc. O desenho observacional utilizado foi nomotético, pontual e de acompanhamento, sendo a unidade de observação a situação de defesa do equipamento observado. Os resultados da análise de qualidade dos dados e generalização mostram índices convincentes para os modelos utilizados. Os resultados da análise das coordenadas polares mostram a maior capacidade de oposição do sistema defensivo 5: 1 de ataque. Destaca também que a transformação de ataque de 3: 3 para 2: 4 é mais usada em face de defesas abertas e provoca o aumento da aparência de sanções disciplinares. A ferramenta de observação projetada e o uso da técnica de coordenadas polares para a estimativa de relações táticas permitem definir procedimentos de intervenção que otimizam os recursos de treinamento e competição no handebol. Palavras chave: Observação sistemática, coordenadas polares, handebol, defesa.

\section{INTRODUCCIÓN}

Dentro de las investigaciones sobre el rendimiento en el deporte, el estudio del comportamiento táctico y la búsqueda de indicadores de desempeño en el entrenamiento y la competición se ha incrementado en los últimos años (Hughes y Franks, 2005; McGarry, O'Donoghue y Sampaio, 2013). En los deportes de equipo, estas pesquisas presentan mayor complejidad para identificar estos indicadores, debido a la velocidad del juego, los cambios constantes de ataque y defensa, el componente de oposición y la dificultad de medir la heterogeneidad de elementos que interactúan para el éxito deportivo (Daza, Andrés y Tarragó, 2017). Esta pluralidad ha contribuido al cambio de consideración en los estudios pasándose de una concepción estática a una dinámica en el juego, un modelo basado en el proceso propugnando estudios de investigación que procuran apreciar las relaciones de interacción y la diversidad del juego en su conjunto (Castellano, 2018; Hugues y Franks, 2005; Liu, Gómez, Lago-Peñas y Sampaio, 2015; Lozano, Camerino e Hileno, 2016; Quiñones et al., 2010; Sampaio, Ibáñez y Lorenzo, 2013; Volossovitz, 2013). Entre los trabajos que poseen el balonmano como referencia (Prieto, Gómez y Sampaio, 2015) hay una proporción asimétrica a favor de los que estudian los aspectos ofensivos con respecto a los defensivos. Probablemente, la dificultad de categorizar la ausencia de balón sea uno de los responsables. Por otro lado, de las investigaciones referidas a la defensa en balonmano, la mayoría estudia términos como eficacia o frecuencia de uso, combinadas con estudios sobre la eficacia en la portería (Rogulj, Shroj y Shroj, 2004). También estudios sobre alternativas o propuestas de aplicación (Espina, 2009, Oliver-Coronado y SosaGonzález, 2016). Escasos son los dedicados a los aspectos colectivos.

Paralelamente, el avance del desarrollo del software especializado ha permitido desarrollar estrategias para el estudio, optimizando la gestión de tiempo y recursos en la investigación con Metodología Observacional. Ésta viene aportando desde hace décadas técnicas de análisis de datos para la obtención de indicadores confiables para el análisis del juego (Anguera y Hernández-Mendo, 2013, 2014, 2015; Lozano y Camerino, 2012; Sequeira, 2012; Sousa, 2014; Sousa, Prudente, Sequeira y Hernández-Mendo, 2014). En este sentido, se hace imprescindible el análisis de la calidad del dato en la validación de las nuevas herramientas diseñadas. A su vez, el análisis de generalizabilidad (TG) (Blanco-Villaseñor, Castellano, Hernández-Mendo, Sánchez-López y Usabiaga, 2014; Cronbach, Gleser, Nanda y Rajaratnam, 1972) estima el grado de generalización del diseño de medida con respecto a las condiciones particulares de un valor teórico buscado. La TG unifica las definiciones de fiabilidad, validez y precisión. Ello permite identificar y medir los 


\section{Coordenadas polares para el estudio de los sistemas defensivos en balonmano}

componentes de variancia que aportan error a una estimación e implementar estrategias que reduzcan su influencia sobre la medida.

En una revisión del rendimiento táctico en deportes de equipo, el análisis de coordenadas polares se muestra como un método emergente de análisis en la literatura especializada (Ávila-Moreno, Chirosa-Ríos, UreñaEspá, Lozano-Jarque y Ulloa-Díaz, 2018). Dicho análisis realiza una reducción drástica de los datos basada en el parámetro Zsum de Cochran (1954), implementada por Sackett (1980) a partir de un análisis secuencial de retardos prospectivos y optimizado con la "técnica genuina" por Anguera (1997) con la perspectiva retrospectiva. De este modo se obtiene una vectorialización del comportamiento y se establecen las relaciones entre una conducta focal y el resto de categorías del sistema. Los valores obtenidos en el cálculo de la probabilidad condicionada permitirán la obtención del parámetro $Z_{\text {sum }}\left(Z_{\text {sum }}=\Sigma \mathrm{z} / \sqrt{ }\right.$ n, siendo $\mathrm{n}$ el número de retardos $)$ (Cochran, 1954). La distribución de este parámetro $Z_{\text {sum }}$ tiene una $\overline{\mathrm{x}}=0$ y una $\mathrm{S}_{\mathrm{x}}=1$. El mapa de coordenadas polares (Gorospe y Anguera, 2000) se obtiene a partir de la obtención de estos valores, siendo necesario determinar el valor de los vectores (iguales o superiores a 1.96 para considerarse significativos) para la elaboración del mismo. El Módulo o longitud del radio se obtiene realizando la raíz cuadrada de la suma del cuadrado de la Zsum de la X (prospectiva) y del cuadrado de la Zsum de la Y (retrospectiva). El ángulo del vector $(\varphi)$ dependerá del cuadrante donde se sitúe y marcará la naturaleza de la relación (Castellano y Hernández-Mendo, 2003). Este ángulo $(\varphi)$ se calcula como $\varphi=$ Arco seno de Y/Radio.

Esta técnica ha sido usada en estudios de diferentes modalidades deportivas, entre las que el balonmano no ha quedado al margen. Así, González, Botejara, Puñales, Trejo y Ruy (2013), analizan la finalización del ataque en partidos igualados; Prudente, Garganta y Anguera (2010) destacan las posibilidades que brinda el análisis secuencial y las coordenadas polares para estudiar las conductas de juego como, por ejemplo, la eficacia del portero valorando la interacción entre portero/compañero y defensor/atacante. Sousa, Prudente, Sequeira, López-López y HernándezMendo (2014) describen patrones de conductas efectivos en las situaciones ofensivas 2 contra 2 , y estos mismos autores (2017) relacionan el duelo 2x2 con la influencia del tiempo y parcial del juego. También se ha usado para examinar la toma de decisiones arbitrales en balonmano (Morillo, Reigal, Hernández-Mendo, Montaña y Morales-Sánchez, 2017). Morillo-Baro, Reigal y Hernández-Mendo (2015) y Navarro, Morillo, Reigal y HernándezMendo (2018) detallan las diferencias entre el juego masculino y femenino en el ataque en balonmano playa. Por su parte, Vázquez-Diz, Morillo-Baro, Reigal, Morales-Sánchez, y Hernández-Mendo (2019a), usan esta técnica para señalar las diferencias de género en la toma de decisiones entre especialistas de balonmano playa y examinan factores contextuales y la toma de decisiones en el ataque posicional en esta misma modalidad (Vázquez-Diz, Morillo-Baro, Reigal, Morales-Sánchez, y Hernández-Mendo, 2019b).

En cuanto al estudio de la defensa en balonmano hay autores que ponderan las acciones defensivas como relevantes al funcionamiento de equipo total (Antúnez, García, Sáez y Valle, 2013; Prudente, 2006; Sáez, Roldán y Feu, 2009; Vuleta, Sporis, Vuleta, Purgar, Herzeg y Milanovic, 2012) o tienen en cuenta la influencia de la eficacia del portero en el rendimiento de los equipos (Pascual, Lago y Casáis, 2010; Prudente et al., 2010). Otros estudios valoran la eficacia defensiva y las variables que influyen en su consecución (Balint y Curiţianu, 2012; Gutiérrez y Férez, 2009). Pero la mayoría de los estudios se ha centrado en parámetros ofensivos, de forma que la presencia de estudios basados en la defensa es escasa con respecto a los basados en indicadores de ataque. De hecho, la Federación Europea de Balonmano no incluye en sus estadísticas la eficacia en acciones defensivas para analizar el rendimiento de forma individual (Skarbalius, Pukénas y Vidūnaité, 2013).

La dificultad de estudiar la defensa proviene por un lado de la ausencia de balón, la dificultad de atribuir o conceptualizar el éxito del comportamiento colectivo y la propia propuesta de un análisis dinámico y complejo. Por lo tanto, el propósito de este estudio ha sido doble, por un lado, diseñar y validar una herramienta que describa las conductas técnicotácticas que manifiestan los jugadores en el desempeño de los sistemas defensivos; y por otro lado, analizar la relación de diferentes comportamientos tácticos ofensivos con las conductas mostradas en los dos principales sistemas defensivos en balonmano a través del análisis de coordenadas polares. 


\section{Jiménez-Salas, Morillo-Baro, Reigal, Morales-Sánchez, Hernández-Mendo}

\section{MATERIAL Y MÉTODOS}

Se ha usado un diseño observacional Nomotético/Puntual/Multidimensional (Anguera, Blanco-Villaseñor, Hernández-Mendo y Losada, 2011) por su eficacia para estudiar conductas de interacción entre deportistas (Anguera y HernándezMendo, 2015). Nomotético, por el análisis diferencial de la pluralidad de los equipos; puntual, al tratarse del registro en una única competición de los equipos, pero con seguimiento intrasesional; y multidimensional, con varias dimensiones que se corresponden con los distintos criterios del instrumento observacional. Se trata de un proceso observacional no participante.

\section{Participantes}

Se han codificado 6988 multieventos, cumpliéndose los requisitos de constancia intersesional, es decir, se elaboró una relación de los menesteres mínimos que permiten caracterizar el perfil de las sesiones de observación que se ajustan al objetivo propuesto. Todo ello con el fin de garantizar el máximo de homogeneidad entre las diferentes sesiones. Se trata de un muestreo intencional donde se observaron cinco partidos de un mismo equipo, el Profasán Mijas frente a cinco diferentes rivales de la $1^{\text {a }}$ división nacional de la competición española de la temporada 2007-08. Número de sesiones de observación propuesto en el estudio de medida del análisis de Generalizabilidad. El consentimiento informado de los deportistas no fue necesario por tratarse de una competición oficial con aforo de público y retransmisiones televisivas.

\section{Instrumentos}

La herramienta de observación ad hoc se ha creado combinando los sistemas de categorías con el formato de campo. Todas las categorías cumplen con el requisito de exhaustividad y mutua exclusividad (Anguera y Mendo, 2013). Dicha herramienta contiene tres macrocriterios: Situación inicial, situación desencadenante y situación final más la duración; 12 criterios: minuto, marcador, equilibrio numérico, sistema defensivo, sistema ofensivo, acción defensiva, zona desencadenante, técnico-táctica individual, medios tácticos, zona finalización, resultado y acción del portero; y 136 categorías. Los criterios, categorías y el sistema de codificación se muestran en la tabla 1 .

Tabla 1. Instrumento de observación de la defensa en balonmano. Criterios y códigos de las categorías que lo componen.

\begin{tabular}{|c|c|c|c|}
\hline CRITERIOS & CATEGORÍAS & CRITERIOS & CATEGORÍAS \\
\hline Minuto & $\begin{array}{l}\text { D05 Inicio hasta minuto } 5 \text {. } \\
\text { D610 Minuto } 6 \text { al } 10 . \\
\text { D1115 Minuto } 11 \text { al } 15 . \\
\text { D1620 Minuto } 16 \text { al } 20 . \\
\text { D2125 Minuto } 21 \text { al } 25 . \\
\text { D2630 Minuto } 26 \text { al } 30 . \\
\text { D3135 Minuto } 31 \text { al } 35 . \\
\text { D3640 Minuto } 36 \text { al } 40 . \\
\text { D4145 Minuto } 41 \text { al } 45 . \\
\text { D4650 Minuto } 46 \text { al } 50 . \\
\text { D5155 Minuto } 51 \text { al } 55 . \\
\text { D5660 Minuto } 56 \text { al } 60 .\end{array}$ & 2. Marcador & $\begin{array}{l}\text { DMPAT Empate. } \\
\text { D12FAV } 1 \text { o } 2 \text { goles a } \\
\text { favor. } \\
\text { DM2FA Más de } 2 \text { goles a } \\
\text { favor. } \\
\text { D12CO } 1 \text { o } 2 \text { goles en } \\
\text { contra. } \\
\text { DM2CO Más de } 2 \text { goles en } \\
\text { contra. }\end{array}$ \\
\hline $\begin{array}{l}\text { 3. } \begin{array}{l}\text { Equilibrio } \\
\text { numérico }\end{array}\end{array}$ & $\begin{array}{l}\text { DIGUA: Igualdad. } \\
\text { D1SUP: uno en superioridad. } \\
\text { DM1SUP: más de uno en sup. } \\
\text { D1INF: uno en inferioridad. } \\
\text { DM1INF: más de uno en inf. }\end{array}$ & $\begin{array}{ll}\text { 4. } & \begin{array}{l}\text { Sistema } \\
\text { defensivo }\end{array}\end{array}$ & $\begin{array}{l}\text { DD321 Defensa 3:2:1 } \\
\text { DD60 Defensa } 6: 0 \\
\text { DD51 Defensa } 5: 1 \\
\text { DD42 Defensa } 4: 2 \\
\text { DD33 Defensa } 3: 3 \\
\text { DD5M1 Defensa } 5+1 \\
\text { DD4M2 Defensa } 4+2 \\
\text { DD50 Defensa } 5: 0 \\
\text { DD40 Defensa doble } \\
\text { inferioridad. } \\
\text { DD41 Defensa } 4: 1 \\
\text { DDIND Defensa individual. }\end{array}$ \\
\hline
\end{tabular}




\section{Coordenadas polares para el estudio de los sistemas defensivos en balonmano}

DREPL Defensa repliegue.

DO33 Ataque contrario 3:3

DO24 Ataque contrario 2:4.

DO32 Ataque contrario 3:2

(en inferioridad).

DO23 Ataque contrario 2:3

5. Sistema ofensivo (en inferioridad).

DOA24 Ataque contrario $3: 3$ al 2:4.

DOCON Contraataque

contrario.

DO22 Ataque contrario 2:2

(doble inferioridad).

DD0 Zona desencadenante 0 .

DD1 Zona desencadenante 1.

DD2 Zona desencadenante 2 .

DD3 Zona desencadenante 3 .

DD4 Zona desencadenante 4.

DD5 Zona desencadenante 5.

DD6 Zona desencadenante 6 .

DD7 Zona desencadenante 7.

DD8 Zona desencadenante 8 .

7. Zona desDD9 Zona desencadenante 9. encadenante

DD10 Zona desencadenante 10.

DD11 Zona desencadenante

11.

DDOCE Zona

desencadenante 12 .

DD13 Zona desencadenante

13.

DD14 Zona desencadenante

14.

DPYVA Pase y va.

DCRPR Cruce o permuta.

DBLPC Bloqueo, pantalla o

corte.

DPENE Penetraciones

sucesivas.

DESP Especiales (fly).

DNOMD Ningún medio.

9. Medios

DCIRC Circulación.

tácticos

colectivos

DDESD Desdoblamiento.

DPCD Pase y va más

circulación o desdoblamiento.

DCRPB Cruce o permuta más bloqueo.

DCPCD Cruce o permuta más circulación o desdoblamiento. DBPCD Bloqueo o pantalla más corte, circulación o desdoblamiento.

DOTCO Otros.
6. Acción

defensiva

8. Técnico-táctica individual
ACOS Acoso.

NACOS No acoso.

CBOP Cambio de oponente.

BLCJ Blocaje.

ROBDE Robo.
DASIT Asistencia.

DCBOP Cruce, bloqueo, permuta.

DPNTR Penetración.

D1X1 Uno contra uno

campo.

DLANZ Lanzamiento.

DLABL Lanzamiento

contra blocaje.

DREB Rebote.

DDSAS Desmarque más asistencia.
10. Zona de finalización
FD0 Zona finalización 0 . FD1 Zona finalización 1. FD2 Zona finalización 2. FD3 Zona finalización 3. FD4 Zona finalización 4. FD5 Zona finalización 5. FD6 Zona finalización 6 . FD7 Zona finalización 7. FD8 Zona finalización 8. FD9 Zona finalización 9. FD10 Zona finalización 10. FD11 Zona finalización 11. FD12 Zona finalización 12. FD13 Zona finalización 13. FD14 Zona finalización 14.

11. Resultado DSANC Amonestación.

12. Portero QUEDA Queda. 


\section{Jiménez-Salas, Morillo-Baro, Reigal, Morales-Sánchez, Hernández-Mendo}

\begin{tabular}{ll}
\hline DPASV Pasivo. & SALTA Salta. \\
DRBT Rebote. & SALSA Sale y salta. \\
DGLSA Gol más sanción. & NADA Nada. \\
D7MFA Penalty fallado. & CAE Cae. \\
DEXCL Exclusión. & SACAE Salta y cae. \\
DDSEX Ataque sin éxito. & \\
DERPR Error pase/recepción. & \\
DGLRE Gol rebote. & \\
DGL7M Gol penalty. & \\
DPGOS Gol penalti más & \\
Sanción. & \\
DNOAR Árbitro para la & \\
jugada. & \\
DRB7M Rebote de penalti. & \\
DPFSA Penalty fallado más & \\
Sanción. & \\
\hline DD12 1 o 2 seg. duración. & \\
DD34 3 o 4 seg. duración. & \\
DD56 5 o 6 seg. duración. & \\
DD78 7 o 8 seg. duración. & \\
DD910 9 o 10 seg. duración. & \\
DD112 11 o 12 seg. duración. & \\
DD14 13 o 14 seg. duración. & \\
DD16 15 o 16 seg. duración. & DD18 17 o 18 seg. duración. \\
DDM20 Más 20 seg. & \\
duración. & \\
\hline
\end{tabular}

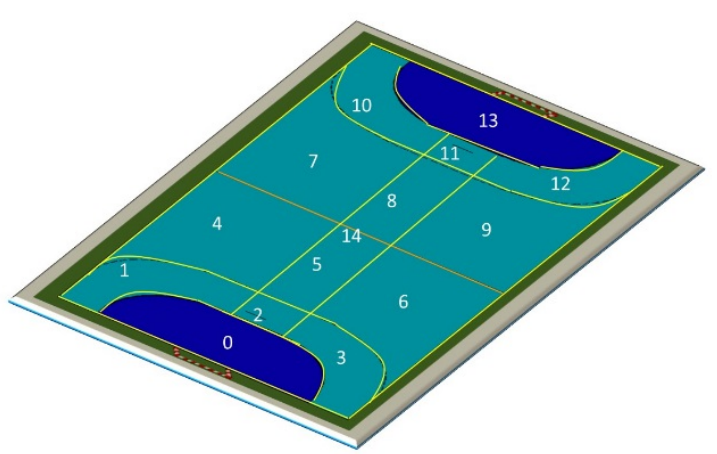

Figura 1 Campograma. Zonas defensivas.

\section{Procedimiento}

Para determinar la fiabilidad de los observadores, identificar las posibles fuentes de error y estimar la calidad de los datos obtenidos a partir de la herramienta de observación, se ha empleado como medida de concordancia la Kappa de Cohen (1960). Previamente se adiestraron en el uso del instrumento dos grupos de dos observadores, todos ellos entrenadores de balonmano. El cálculo se ha realizado mediante el programa informático GSEQ, versión 1.0., teniendo en consideración las recomendaciones de Bakeman y Quera (2011), obteniéndose valores de 0.85 correspondiente a los grupos de conductas pertenecientes a la situación final, 0.89 para los de la situación desencadenante y 1 para los de la situación final, por lo que pueden ser considerados como excelentes. Asimismo, son óptimos los resultados del análisis de Generalizabilidad, obtenidos mediante el programa informático SAGT 1.0. (Hernández-Mendo, Ramos-Pérez y Pastrana, 2012) que muestra una fiabilidad de 0.99 , tanto intra como interobservadores. Se emplearon dos partidos. La optimización del número mínimo de partidos expresó un índice de 0.973 para cinco partidos analizados.

Para el análisis de datos se han empleado las Coordenadas Polares, que viene siendo usada como línea de trabajo para la estimación de interrelaciones en contextos deportivos (Castellano y HernándezMendo, 2003; Díaz, Hernández-Moreno y HernándezFlores, 2016; Hernández-Mendo y Anguera, 1998; Gorospe, 1999) y en particular en balonmano (González, Botejara, Puñales, Trejo y Ruy, 2013; Sousa, Prudente, Sequeira, López-López y Hernández-Mendo, 2015). Esta técnica desarrollada por Sackett (1980) y optimizada por Anguera (1997) 


\section{Coordenadas polares para el estudio de los sistemas defensivos en balonmano}

permite comprobar la intensidad de la relación entre una conducta focal (condicionante) y el resto de conductas (condicionadas) propuestas en el sistema taxonómico. A partir de ahí se construye un mapa vectorial (Prudente, 2006) que permite interpretar la índole de las interrelaciones en función del cuadrante donde se ubique el vector y el ángulo y radio resultantes.

Las conductas seleccionadas como focales han sido las principales defensas usadas (5:1, DD51 y 6:0, DD60) y los principales sistemas ofensivos usados contra estas defensas (3:3, DO33 y 2:4, DO24). Como conductas de apareo las categorías de la zona desencadenante y de finalización de la acción ofensiva, la técnico-táctica individual ofensiva usada, los medios tácticos básicos colectivos, la acción defensiva elicitada y el resultado de la acción.

Para el análisis se ha usado el programa HOISAN, v.1.6 (Hernández-Mendo, López-López, Castellano,
Morales-Sánchez y Pastrana, 2012; HernándezMendo et al., 2014).

\section{RESULTADOS}

La técnica de coordenadas polares ha permitido encontrar patrones de conducta entre las conductas focales seleccionadas y el resto de conductas de la herramienta de observación.

Los resultados del análisis de coordenadas polares tomando como conductas focales los principales sistemas de juego defensivos se muestran en la representación vectorial de la figura 2 , así como en la tabla 2 .

Figura 2. Representación del plano vectorial de las defensas 5:1 y 6:0
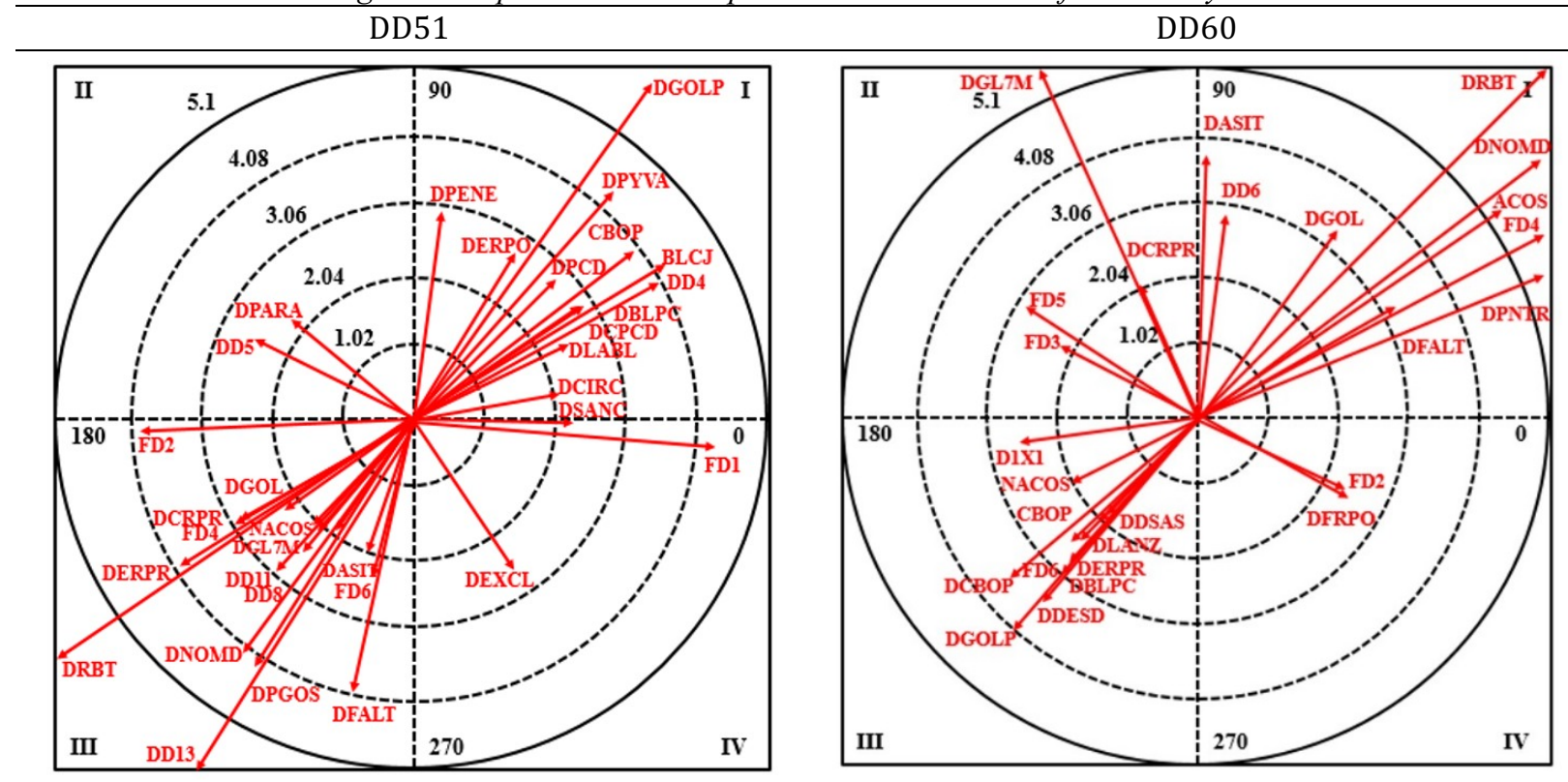

Tabla 2. Muestra de asociaciones significativas entre la conducta focal y las de apareo en defensas 5:1 y 6.0

\begin{tabular}{ccccccc}
\hline C & \multicolumn{3}{c}{ DD51 } & & \multicolumn{3}{c}{ DD60 } \\
\cline { 2 - 7 } & $\begin{array}{c}\text { Cond. } \\
\text { Apareo }\end{array}$ & R & Ángulo & $\begin{array}{c}\text { Cond. } \\
\text { Apareo }\end{array}$ & R & Ángulo \\
\hline \multirow{3}{*}{ I CBOP } & 3.81 & 34.48 & ACOS & 6.79 & 26.84 \\
\cline { 2 - 7 } & BLCJ & 4.13 & 29.68 & DD6 & 2.74 & 83.39 \\
\hline & DD4 & 4.06 & 22.61 & DASIT & 3.74 & 88.9 \\
\hline
\end{tabular}

\begin{tabular}{lrrlrr} 
DLABL & 2.58 & 21.64 & DPNTR & 5.69 & 18.3 \\
\hline DPYVA & 4.41 & 48.82 & DNOMD & 5.47 & 32.56 \\
\hline DBLPC & 2.81 & 30.79 & FD4 & 8.77 & 23.25 \\
\hline DPENE & 2.94 & 81.37 & DGOL & 3.38 & 52.75 \\
\hline DCIRC & 2.1 & 6.82 & DFALT & 3.25 & 23.96 \\
\hline DPCD & 2.7 & 45 & DRBT & 8.02 & 45.01 \\
\hline DCPCD & 2.77 & 32.53 & & &
\end{tabular}




\section{Jiménez-Salas, Morillo-Baro, Reigal, Morales-Sánchez, Hernández-Mendo}

\begin{tabular}{|c|c|c|c|c|c|c|}
\hline & DFRPO & 2.79 & 62.58 & & & \\
\hline & DGOLP & 5.87 & 58.44 & & & \\
\hline \multirow{4}{*}{ II } & DD5 & 2.47 & 166.07 & DCRPR & 2.09 & 105.23 \\
\hline & DPARA & 2.14 & 143.36 & FD3 & 2.25 & 154.98 \\
\hline & & & & FD5 & 2.95 & 150.37 \\
\hline & & & & DGL7M & 2.62 & 116.17 \\
\hline \multirow{16}{*}{ III } & NACOS & 2.2 & 226.06 & NACOS & 5.45 & 201.9 \\
\hline & DD8 & 3.03 & 231.83 & CBOP & 2.46 & 224.56 \\
\hline & DD11 & 3.83 & 224.98 & DCBOP & 3.63 & 220.39 \\
\hline & DD13 & 7.11 & 241.68 & D1X1 & 2.45 & 188.43 \\
\hline & DASIT & 2 & 254.16 & DLANZ & 2.3 & 225 \\
\hline & DCRPR & 2.82 & 208.91 & DDSAS & 1.98 & 225 \\
\hline & DNOMD & 3.16 & 232.67 & DBLPC & 3.1 & 225 \\
\hline & FD2 & 3.84 & 182.77 & DDESD & 3.45 & 225 \\
\hline & FD4 & 3.06 & 209.18 & FD6 & 2.89 & 224.92 \\
\hline & FD6 & 2.27 & 266.46 & DGOLP & 4.04 & 224.9 \\
\hline & DGOL & 2.21 & 219.29 & DERPR & 2.09 & 225 \\
\hline & DFALT & 4.05 & 258.76 & & & \\
\hline & DRBT & 5.57 & 217.59 & & & \\
\hline & DERPR & 3.97 & 213.13 & & & \\
\hline & DGL7M & 2.5 & 220.5 & & & \\
\hline & DPGOS & 3.19 & 237.99 & & & \\
\hline \multirow{3}{*}{ IV } & FD1 & 4.35 & 351.63 & FD2 & 2.7 & 351.29 \\
\hline & DSANC & 2.29 & 359.75 & DFRPO & 2.74 & 349.81 \\
\hline & DEXCL & 2.56 & 310.84 & & & \\
\hline
\end{tabular}

En la tabla 2, y en lo que respecta al cuadrante I donde se encuadran las acciones con una relación mutuamente excitatoria destacan las categorías: Cambio de oponente CBOP y blocaje BLCJ del criterio acción defensiva con la defensa 5:1; mientras que con la defensa 6:0 y este mismo criterio, aparece el acoso ACOS.

Por lo que respecta al criterio acción técnico-táctica individual ofensiva, es reseñable que la conducta focal defensa 6:0 encuentra relaciones significativas con la categoría asistencia DASIT y la penetración DPNTR. En cuanto a la zona de finalización aparece una intensa relación con FD4, delimitada entre los $9 \mathrm{~m}$. y el centro del campo, en cuanto a profundidad, y la zona de fuera de banda y la perpendicular del poste izquierdo de la portería, en cuanto a la anchura.

Varias son las categorías del criterio medios tácticos colectivos ofensivos que subrayan esta relación

significativa con la defensa 5:1 como son: Pase y va DPYVA, Bloqueo, Pantalla o corte DBLPC, penetraciones sucesivas DPENE, Circulación DCIRC, y las combinaciones del pase y va y el cruce y permuta con circulación/desdoblamiento DPCD y DCPCD.

Dentro del macrocriterio resultado, se acentúan las relaciones entre la conducta focal defensa 5:1 y el golpe franco DGOLP. Mientras que en la defensa 6.0 es con la falta técnica la categoría asociada significativamente.

También se encuentran asociaciones significativas en el cuadrante II, donde la conducta criterio es inhibitoria y las conductas de apareo son excitatorias. Cuando la defensa 6:0 es inhibitoria, aparecen varias conductas excitatorias, siendo reseñable la del cruce o permuta DCRPR.

Del mismo modo y ya en el cuadrante III, donde la conducta criterio y la conducta de apareo son mutuamente inhibitorias, encontramos relaciones entre la defensa 6:0 y el cambio de oponente CBOP.

Por lo que respecta a la acción técnico-táctica individual y la defensa 6:0 se relaciona con la acción 1x1 D1X1.

Respecto a los medios tácticos colectivos, se matiza que la defensa 5:1 muestra relaciones con el cruce y permuta DCRPR y la defensa 6:0 con el bloqueo, pantalla o corte DBLPC y el desdoblamiento DDESD. Por último, en lo que concierne al resultado, se recalca que la defensa 5:1 muestra relaciones significativas con la falta técnica DFALT y la defensa 6:0 se relaciona con el golpe franco DGOLP.

En el cuadrante IV la conducta criterio es excitatoria y la conducta de apareo es inhibitoria. De las relaciones halladas en el cuadrante IV relacionadas con la defensa 5:1 se acentúan los resultados de amonestación y exclusión. En el mismo cuadrante, pero relacionadas con la defensa 6:0 destaca el resultado de fuera o poste DFRPO.

Los resultados del análisis de coordenadas polares tomando como conductas focales los principales sistemas de juego ofensivos se muestran en la representación vectorial de la figura 3 , así como en la tabla 3 . 


\section{Coordenadas polares para el estudio de los sistemas defensivos en balonmano}

Figura 3. Representación del plano vectorial de los sistemas de ataque en desdoblamiento a 2:4 y 3:3 DOA24
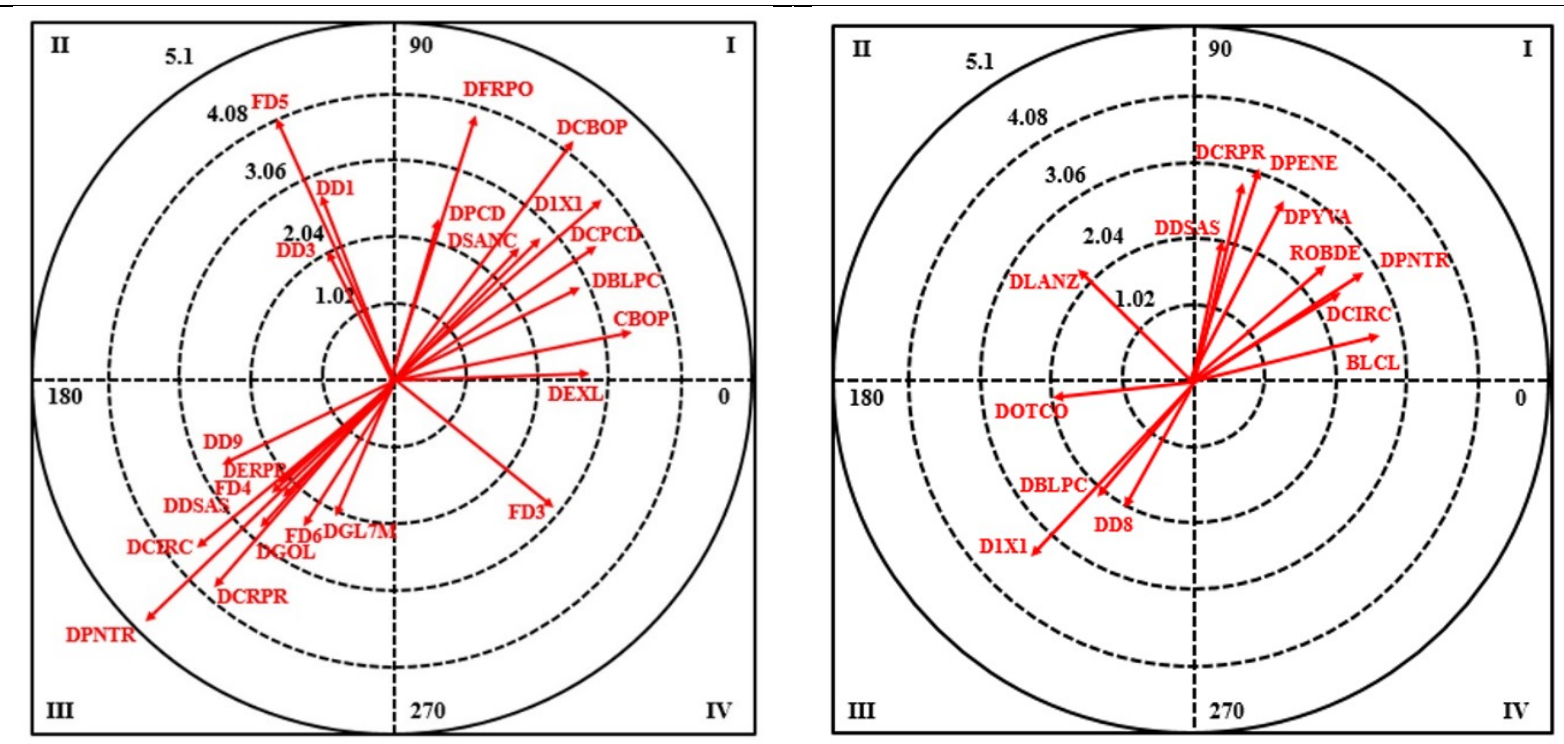

Tabla 3. Muestra de asociaciones significativas entre la conducta focal y las de apareo en los ataques 3:3 y desdoblamiento a 2:4

\begin{tabular}{|c|c|c|c|c|c|c|}
\hline \multirow[b]{2}{*}{$\mathrm{C}$} & \multicolumn{3}{|c|}{ DOA24 } & \multicolumn{3}{|c|}{ DO33 } \\
\hline & $\begin{array}{c}\text { Cond. } \\
\text { Apareo }\end{array}$ & $\mathbf{R}$ & Ángulo & $\begin{array}{c}\text { Cond. } \\
\text { Apareo }\end{array}$ & $\mathbf{R}$ & Ángulo \\
\hline \multirow{9}{*}{ I } & CBOP & 3,32 & 9,45 & BLCJ & 2,67 & 10,66 \\
\hline & DCBOP & 4,13 & 56,09 & ROBDE & 2,4 & 37,08 \\
\hline & D1X1 & 3,77 & 37,04 & DPNTR & 2,74 & 27,87 \\
\hline & DBLPC & 2,82 & 20,77 & DPYVA & 2,73 & 68,48 \\
\hline & DPCD & 2,3 & 78,29 & DCRPR & 2,82 & 82,63 \\
\hline & DCPCD & 3,31 & 26,08 & DPENE & 3,06 & 76,88 \\
\hline & DFRPO & 3,89 & 77,46 & DCIRC & 2,48 & 26,67 \\
\hline & DSANC & 2,45 & 48,85 & DDSAS & 1,99 & 84,67 \\
\hline & DEXCL & 2,74 & 0,84 & & & \\
\hline \multirow{3}{*}{ II } & DD1 & 2,7 & 104,74 & DLANZ & 2,35 & 135,34 \\
\hline & DD3 & 2,05 & 126,58 & & & \\
\hline & FD5 & 4,09 & 119,54 & & & \\
\hline III & DD9 & 2,69 & 202,83 & DD8 & 2,05 & 243,78 \\
\hline
\end{tabular}

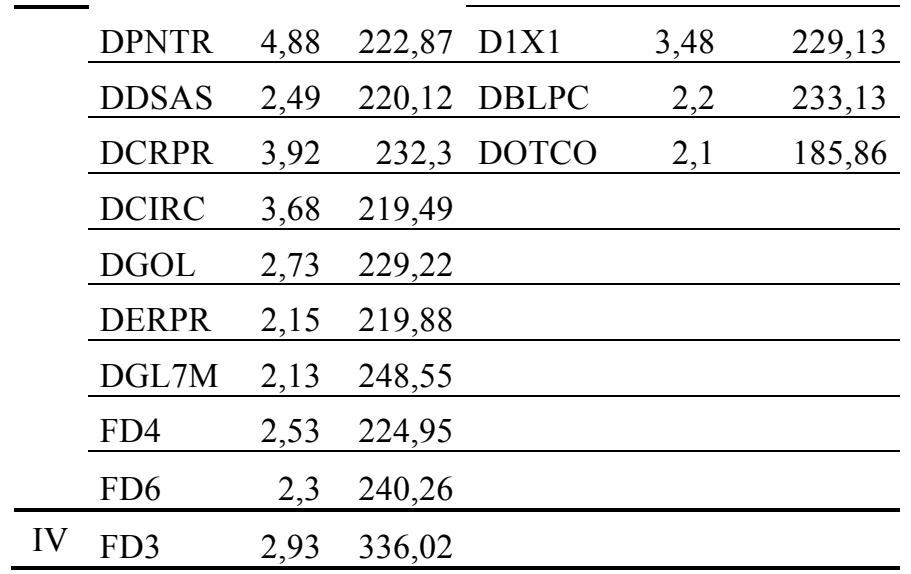

Con respecto a los dos sistemas de ataque estudiados, el primer cuadrante el ataque 3:3 muestra una relación mutuamente excitatoria con las acciones de técnicotáctica individual: penetración DPNTR y desmarque más asistencia DDSAS, las acciones defensivas blocaje BLCJ y robo ROBDE, los medios tácticos colectivos cruce o permuta DCRPR, las penetraciones sucesivas DPENE y la circulación DCIRC. 


\section{Jiménez-Salas, Morillo-Baro, Reigal, Morales-Sánchez, Hernández-Mendo}

Por su parte, el sistema 3:3 a 2:4 lo hace con la acción defensiva cambio de oponente CBOP, con las acciones de técnico táctica individual: cruce, bloqueo o permuta DCBOP y la acción uno contra uno 1X1, los medios tácticos: bloqueo, pantalla o corte DBLPC, el pase y va más circulación o desdoblamiento DPCD y el cruce o permuta CDPCD.

El ataque $3: 3$ a 2:4 presenta asimismo relaciones mutuamente excitatorias con el resultado sanción DSANC y la exclusión DEXCL y los marcadores a favor D12FA o claramente en contra DM2CO.

En el cuadrante III, la conducta criterio $3: 3$ se relaciona de forma mutuamente inhibitoria con las siguientes categorías: Uno contra uno $1 \mathrm{x} 1 \mathrm{y}$ bloqueo, pantalla o corte DBLPC. La conducta criterio DOA24 lo hace con las acciones defensivas penetración DPNTR y desmarque más asistencia DDSAS, los medios cruce o permuta DCRPR y circulación DCIRC, y el resultado error de pase/recepción DERPR.

\section{DISCUSIÓN}

El propósito de esta investigación ha sido describir y analizar patrones de conducta y las relaciones establecidas entre los principales tipos de defensa, medios tácticos defensivos usados y la posible relación de flujo comportamental con los comportamientos tácticos empleados por el contrario, utilizando la práctica de coordenadas polares (Prudente, Sousa, Sequeira, López-López y Hernández-Mendo, 2017; Navarro, Morillo, Reigal y Hernández-Mendo, 2018).

El estudio ha confirmado que la herramienta observacional posee unas estimaciones de fiabilidad y coeficientes de correlación con valores adecuados. El análisis de Generalizabilidad muestra una alta precisión de medida. También queda garantizada la adecuación del sistema de observación y la capacidad de generalización de los resultados obtenidos. El software HOISAN (Hernández-Mendo, López-López, Castellano, Morales-Sánchez y Pastrana, 2012; Hernández-Mendo et al., 2014) ha posibilitado la formulación de cálculos para las coordenadas polares, así como su representación vectorial. Asimismo, se constata que esta técnica ha permitido identificar asociaciones significativas entre comportamientos tácticos defensivos y comportamientos condicionales. En cuanto al análisis e interpretación de resultados propios del juego, aparecen relaciones de mutua excitación entre las conductas focales seleccionadas y las conductas de apareo. Estas vinculaciones, señalan orientaciones diferentes según la defensa escogida.

Dentro de las posibilidades de la herramienta creada y validada, se han escogido como conductas focales las defensas 5:1 y 6:0 por ser las más usadas en balonmano (Prudente, 2006; Silva, 2008 y Sequeira 2012). Así, en la defensa 6:0 aparecen asociaciones manifiestas con el uso del acoso defensivo. De hecho, aunque tradicionalmente se considera la defensa 6:0 como una defensa cerrada, esta denominación se corresponde con su punto de partida, pero el hecho de evitar lanzamientos cómodos por parte del contrario o aumentar su grado de oposición justifica la alta significatividad entre ambas conductas. Por su parte, el ataque usa las penetraciones y asistencias como táctica individual con preferencia sobre los medios tácticos.

Por su parte, en la defensa 5:1 dichas asociaciones se manifiestan con el blocaje y el cambio de oponente como acciones defensivas. Los medios tácticos básicos colectivos son la circulación, el pase y va y el desdoblamiento, así como el cruce y la permuta. También aparece significatividad con las sanciones de golpe franco. Coinciden los resultados con los de Sequeira (2012) que postula que la defensa 5:1 presenta mayor capacidad de oposición. Aunque Lozano, Camerino e Hileno (2016) coinciden en que los equipos ganadores hallan medios tácticos adecuados para resolver las exigencias defensivas en momentos críticos de juego, contra la defensa 5:1.

El ataque con desdoblamiento (DOA24) se vincula con la defensa 5:1, con los cambios de oponente defensivo y con el cruce, bloqueo y $1 \mathrm{x} 1$ como medios tácticos individuales usados por el ataque. Tiene una fuerte relación con marcador a favor o claramente en contra, lo que sugiere que no suele ser esta defensa la usada como primera opción, sino más bien como recurso. De hecho, Lozano, Camerino e Hileno (2016), encuentran como T-pattern más relevante en los equipos ganadores en momentos críticos de juego la secuencia de ataque posicional ante defensa 5:1. También atañe a la aparición de sanción o exclusión 


\section{Coordenadas polares para el estudio de los sistemas defensivos en balonmano}

del contrario. De hecho, cuando la defensa se establece en varias líneas para dificultar los lanzamientos a distancia, aumenta al mismo tiempo la dificultad de establecer el cambio de oponente, por el propio hecho que los defensores se encuentran en varias líneas. De ahí que el ataque adapte sus comportamientos ofensivos buscando la circulación y desdoblamiento de jugadores, así como los cambios de puesto ofensivos mediante los cruces o permutas.

El ataque 3:3 revela relaciones específicas con situaciones de marcador desfavorable o empate, tanto ante defensa 5:1 como 6:0. De hecho, aunque la situación de ataque culmine en un sistema 2:4, suele ser por desdoblamiento de algún jugador, y rara vez se parte de esta configuración ofensiva. Esta conducta focal afecta de manera significativa a acciones defensivas como blocajes y robos y ataques con desmarque más asistencia y medios tácticos como pase y va, penetraciones sucesivas y circulaciones. Las finalizaciones se producen en la zona izquierda de la defensa (FD4) probablemente por la tendencia de los jugadores atacantes diestros de salir hacia la derecha, su punto fuerte.

Las diferencias entre conductas no se producen sólo en la relación excitatoria ya que aparece una relación mutuamente inhibitoria entre cruce, permuta $y$ provocación de falta técnica como conductas de apareo y la defensa 5:1 como conducta focal. Esa misma relación aparece entre cambio de oponente, bloqueos, 1x1 y golpe franco con la defensa 6:0.

En definitiva, este trabajo aporta una interpretación del juego útil para el entrenamiento y destaca la importancia del análisis de la competición desde una concepción dinámica y cualitativa, estudiando variables sobre comportamientos tácticos $\mathrm{y}$ situacionales (O’Donoghue, 2005; Prieto, Gómez y Sampaio, 2015).

Ha resultado una dificultad analizar las conductas en defensa debido a la complejidad de delimitar responsabilidades, como se comenta en el estudio. Además de tener que centrar el mismo en dos cuestiones específicas, cuando el estudio de un sistema defensivo es más complicado. Por ejemplo, por zonas, puestos específicos en defensa, duración de la situación.

Por otro lado, ha supuesto una limitación dejar fuera del estudio sistemas otros sistemas defensivos, como los producidos en situaciones de desigualdad numérica que no han sido analizados.

\section{CONCLUSIONES}

Una vez más esta técnica se ha mostrado eficaz como herramienta para aflorar e interpretar las complejas relaciones comportamentales entre diferentes fases del juego en balonmano, aportando una importante información a los entrenadores que les permitirá intervenir con los deportistas en la mejora del rendimiento (Prudente, Sousa, Sequeira, López-López y Hernández-Mendo, 2017). Se han distinguido los diferentes medios tanto individuales como colectivos que usan los equipos atacantes ante los sistemas defensivos propuestos y qué medios defensivos son los más usados y con mayor eficacia para evitar la consecución del gol.

\section{APLICACIONES PRÁCTICAS}

Este estudio aporta información al entrenador sobre las diferentes opciones tácticas que usan los equipos ante los sistemas defensivos planteados. Ello da la oportunidad de aplicar de forma previa durante el entrenamiento las medidas para contrarrestar la táctica del contrario. Así se deduce la importancia de mejorar la competencia defensiva en 6:0 ante penetraciones y asistencias y procurar una respuesta eficaz ante situaciones de circulación y desdoblamientos; así como los cruces y permutas desde la defensa 5:1.

\section{REFERENCIAS}

1. Anguera, M.T. (1997). From prospective patterns in behavior to joint analysis with a retrospective perspective. Colloque sur invitation «Méthodologie d'analyse des interactions sociales». Université de la Sorbonne. Paris.

2. Anguera, M.T. y Hernández-Mendo, A. (2013). La metodología observacional en el ámbito del deporte. E-balonmano.com: Revista de Cienc. del Deporte 9(3), 135-160.

3. Anguera, M.T. y Hernández-Mendo, A. (2014). Metodología observacional y psicología del deporte: Estado de la cuestión. Revista de Psicol. del Deporte, 23(1), 103109.

4. Anguera, M.T. y Hernández-Mendo, A. (2015). Técnicas de análisis en estudios observacionales en ciencias del deporte. Cuadernos de Psicología del Deporte, 15(1), 13-30. 


\section{Jiménez-Salas, Morillo-Baro, Reigal, Morales-Sánchez, Hernández-Mendo}

https://doi.org/10.4321/S157884232015000100002

5. Anguera, M.T., Blanco, A., HernándezMendo, A., y Losada, J. L. (2011). Diseños observacionales: ajuste y aplicación en psicología del deporte. Cuad. de Psicol. del Deporte, 11(2), 63-76. http://dx.doi.org/10.4321/S157884232015000100002

6. Antúnez, A., García, J., Sáez, F., Valle, A., y García, A. (2013). Diferencias en los indicadores de rendimiento entre los equipos ganadores y perdedores en etapas de formación en balonmano en función del género y la diferencia final de goles. E-Balonmano, 9(1), $5-16$.

7. Ávila-Moreno, F.M., Chirosa-Ríos, L.J., UreñaSpá, A., Lozano-Jarque, D., y Ulloa-Díaz, D. (2018). Evaluation of tactical performance in invasion team sports: a systematic review, International Journal of Performance Analysis in Sport, 18:2, 195-216, https://doi.org/10.1080/24748668.2018.1460054

8. Bakeman, R. y Quera, V. (2011). Sequential analysis and observational methods for the behavioral sciences. Cambridge: Cambridge University Press. https://doi.org/10.1017/CBO9781139017343

9. Balint, E., Curitianu, I. (2012). The importance of anticipation in increasing the defense efficiency in high performance handball. Bulletin of the Transilvania University of Braşov. Series VIII: Art • Sport • Vol. 5 (54). https://doi.org/10.1016/j.sbspro.2013.04.077

10. Blanco-Villaseñor, A., Castellano, J., HernándezMendo, A., Sánchez-López, C. R. y Usabiaga, O. (2014). Aplicación de la TG en el deporte para el estudio de la fiabilidad, validez y estimación de la muestra. Revista de Psicología del Deporte, 23(1), 131-137.

11. Castellano, J. (2018). Relación entre indicadores de rendimiento en el fútbol profesional. Revista iberoamericana de psicología del ejercicio y el deporte 13(1), 41-49.

12. Castellano, J. y Hernández-Mendo, A. (2003): El análisis de coordenadas polares para la estimación de relaciones en la interacción motriz en fútbol. Psicothema, 15(4), 569-579.

13. Cochran W. G. (1954). Some methods for streghning the common $\chi^{2}$ test. Biometrics, 10, 417-451. doi: $10.2307 / 3001616$

14. Cohen, J. (1960). A coefficient of agreement for nominal scales. Educational and Psychological Measurement, 20, 37-46. https://doi.org/10.1177/001316446002000104

15. Cronbach, L. J., Gleser, G. C., Nanda, H. y Rajaratnam, N. (1972). The dependability of behavioral measurements: theory of generalizability for scores and profiles. Nueva York: John Wiley and Sons.

16. Daza, G.; Andrés, A., y Tarragó, R. (2017). Match Statistics as Predictors of Team's Performance in elite competitive Handball. RICYDE. Revista internacional de ciencias del deporte, 48(13), 149-161.

https://doi.org/10.5232/ricyde2017.04805

17. Díaz, R., Hernández-Moreno. J. y HernándezFlores, C. N. (2016). Análisis de las interacciones motrices en fútbol a través de coordenadas polares. Acción Motriz n ${ }^{0}$ 16. Asociación Científico Cultural en Actividad Física y Deporte. (ACCAFIDE) Las Palmas de Gran Canaria.

18. Espina, J. J. (2009). Evolución histórica, táctica y estructural de los sistemas de juego defensivos en balonmano. Una aplicación en la educación superior. Tesis doctoral. Universidad de Alicante. Alicante.

19. González A., Botejara, J., Puñales, L., Trejo, A. y Ruy, E. (2013). Análisis de la finalización del ataque en partidos igualados en balonmano de alto nivel mediante coordenadas polares. E-balonmano.com: Revista de Ciencias del Deporte 9(2), 71- 89.

20. Gorospe, G. (1999). Observación y análisis en el tenis de individuales. Aportaciones del análisis de secuencialidad y de las coordenadas polares. Universidad del País Vasco: Tesis Doctoral.

21. Gorospe, G. y Anguera, M.T. (2000). Modificación de la técnica clásica de coordenadas polares mediante un desarrollo 


\section{Coordenadas polares para el estudio de los sistemas defensivos en balonmano}

distinto de la retrospectividad: aplicación al tenis. Psicothema, 12(2), 279-282.

22. Gutiérrez, O. y Férez, J.A. (2009). Cuantificación y valoración de la eficacia de los sistemas defensivos empleados en el marco situacional de igualdad numérica en los equipos de balonmano de alto nivel. Cronos. Rendimiento en el deporte. VIII, 14, 113-116.

23. Hernández-Mendo, A. y Anguera, M.T. (1998). Análisis de coordenadas polares en el estudio de las diferencias individuales de la acción de juego. En: Sánchez, M. P., LópezQuiroga Estévez, M.A. (eds.). Perspectivas actuales en la investigación de las diferencias individuales, (pp. 84-88). Centro de Estudios Ramón Areces, Madrid.

24. Hernández-Mendo, A., Castellano, J., Camerino, O., Jonsson, G., Blanco-Villaseñor, A., Lopes, A. y Anguera, M. T. (2014). Programas informáticos de registro, control de calidad del dato, y análisis de datos. Revista de Psicol. del Deporte, 23(1), 111-121.

25. Hernández-Mendo, A., López López J.A., Castellano, J., Morales-Sánchez, V. y Pastrana, J.L. (2012). Hoisan 1.2: Programa informático para uso en metodología observacional. Cuad. de Psicol. del Deporte, $12(1), \quad 55-78$. doi.org/10.4321/S1578$\underline{84232012000100006}$

26. Hernández-Mendo, A., Ramos-Pérez, F. y Pastrana, J. L. (2012). SAGT: Programa informático para análisis de Teoría de la Generalizabilidad. SAFE CREATIVE Código: 1204191501059.

27. Hughes, M. y Franks, I. (2005). Analysis of passing sequences, shots and goals in soccer. Journal of Sports Sciences, 23(5), 509-514. https://doi.org/10.1080/02640410410001716779

28. Liu, H., Gomez, M.A., Lago-Peñas, C. y Sampaio, J. (2015). Match statistics related to winning in the group stage of 2014 Brazil FIFA World Cup. Journal of sports sciences, 33(12), 1205-1213.

https://doi.org/10.1080/02640414.2015.1022578

29. Lozano, D. y Camerino, O. (2012). Eficacia de los sistemas ofensivos en balonmano. Apunts,
$108, \quad 70-81$ doi.org/10.5672/apunts.20140983.es. $(2012 / 2) .108 .08$

30. Lozano, D., Camerino, O. y Hileno, R. (2016). Análisis del comportamiento táctico ofensivo en momentos críticos de juego en el alto rendimiento en balonmano: un estudio Mixed Methods. Cuadernos de Psicología del Deporte 16(1) pp: 151-160. ISSN 1989-5879

31. McGarry, T., O'Donoghue, P. y Sampaio, J. (2013). Routledge handbook of sports performance analysis. London: Routledge. https://doi.org/10.4324/9780203806913

32. Morillo-Baro, J.P., Reigal, R.E. y HernándezMendo, A. (2015). Análisis del ataque posicional de balonmano playa masculino y femenino mediante coordenadas polares. RICYDE. Revista Int. de Cienc. del Deporte. 11(41), 226-244. doi.org/10.5232/ricyde2015.04103

33. Morillo, J.P., Reigal, RE, Hernández-Mendo, A., Montaña, A. y Morales-Sánchez, V. (2017). Toma de decisiones por árbitros de balonmano: diseño de un instrumento de observación ad hoc y análisis de coordenadas polares. Frontiers in Psychology, 8, doi.org/10.3389/fpsyg.2017.01842

1842.

34. Navarro, A, Morillo, J.P., Reigal, J. E. y Hernández-Mendo, A. (2018). Polar coordinate analysis in the study of positional attacks in beach handball, International Journal of Performance Analysis in Sport, 18:1, 151167, DOI: $10.1080 / 24748668.2018 .1460052$

35. O'Donoghue, P. (2005). Normative Profiles of Sports Performance. International Journal of Performance Analysis in Sport, 5(1), 104119(16). https://doi.org/10.1080/24748668.2005.11868319

36. Oliver-Coronado, F.J. y Sosa González, P. I. (2016). Los sistemas defensivos en balonmano y la complejidad: su entrenamiento desde el error. IX Congreso Int. Asoc. Esp. Cienc. del Deporte. Facultad de Cienc. del deporte. Univ. de Castilla-La Mancha.

37. Pascual Fuertes, X., Lago Peñas, C. y Casáis Martínez, L. (2010). La influencia de la eficacia del portero en el rendimiento de los equipos de 


\section{Jiménez-Salas, Morillo-Baro, Reigal, Morales-Sánchez, Hernández-Mendo}

balonmano. Apunts: Educación física $y$ deportes, 99, 72-81.

38. Prieto, J., Gómez, M.A. y Sampaio, J. (2015). From a static to a dynamic perspective in handball match analysis: A systematic review. The open sports sci. j., $8, \quad 25-34$. https://doi.org/10.2174/1875399X01508010025

39. Prudente, J. (2006). Análise da performance táctico-técnica no andebol de alto nivel. Estudo das acçoes ofensivas com recurso à análise sequencial. Dissertaçao apresentada com vista a obtençao do grau de Doutor em Educaçao Física e Desporto. Univ. da Madeira.

40. Prudente, J., Garganta, J. y Anguera, M. T. (2010). Methodological Approach to evaluate interactive behaviors in team games: An example in handball. En Proc. of meas. behav. Eindhoven, Netherland. https://doi.org/10.1145/1931344.1931385

41. Prudente, J., Sousa, D., Sequeira, P., LópezLópez, J., y Hernández-Mendo, A. (2017). Analyzing the influence of playing time and partial score on the tactical behavior in the attack in handball, using the polar coordinates technique. Anales de Psicol. 33(3), 515-529 doi.org/10.6018/analesps.33.3.271071

42. Quiñones, Y., Morillo-Baro, J.P., Reigal, R.E., Morales-Sánchez, V., Vázquez-Diz, J.A. y Hernández-Mendo, A. (2020). El juego combinativo ofensivo en el balonmano de élite: diferencias por género mediante análisis de coordenadas polares. Cuadernos de psicología del deporte. En prensa.

43. Rogulj, N., Shroj, V. y Shroj, L. (2004). The Contribution of Collective Attack Tactics in Differentiating Handball Score Efficiency. Coll. Antropol. 28, 2: 739-746.

44. Sáez, F. J., Roldán, A. y Feu, S. (2009). Diferencias en las estadísticas de juego entre los equipos ganadores y perdedores de la copa del rey 2008 de balonmano masculino. $E$ balonmano.com: Revista de Ciencias del Deporte, 5(3), 107-114.

45. Sampaio J., Ibáñez S. y Lorenzo A. (2013). Basketball. In: McGarry T, O'Donoghue P, Sampaio J, Eds. Routledge handbook of sports performance analysis. Abingdon, UK: Routledge 2013; pp. 357-66

46. Sackett, G.P. (1980). Lag Sequential Analysis as a data Reduction Technique in Social Interaction Research. En D.B. Sawin, R.C. Hawkins, L.O. Walker and J.H. Penticuff (Eds.), Except. infant. Psychosocial risks in infant-environ. trans. (pp. 300-340). Brunner/Mazel, New York

47. Sequeira, A. (2012). Análisis de los Factores de Eficacia de las Acciones de Pre-finalización y Finalización en Ataque Organizado en el Balonmano de Alto Nivel. (Tesis Doctoral). FCD - Universidad de Castilla-La Mancha, Toledo.

48. Skarbalius, A.; Pukénas, K. y Vidunaité, G. (2013). Sport performance profile in men's European modern handball: discriminant analysis between winners and losers. Ugdymas, Kuno Kultura, Sportas, 3(90), 44-54.

49. Silva, J. (2008). Modelação Táctica do Processo Ofensivo em Andebol. Estudo de situações de igualdade numérica, 7 vs 7 , com recurso à Análise Sequencial. (Tesis doctoral). Universidade do Porto, Porto.

50. Sousa, D. (2014). Estudo das Situações de Jogo 2 vs 2 Ocorridas em Ataque Organizado em Situação de Igualdade Numérica 6 vs 6 , no Andebol de Alto Nível Masculino e Feminino Análise do Campeonato da Mundo 2011 de Seniores Femininos e do Campeonato da Europa 2012 de Seniores Masculinos, com recuso à análise sequencial. (Tese de Doutoramento não publicada). Universidade da Madeira, Funchal.

51. Sousa, D., Prudente, J., Sequeira, P. y Hernández-Mendo, A. (2014). Análise da Qualidade dos Dados de um Instrumento para Observação do 2vs2 no Andebol. in Revista Iberoamericana de Psicologia del Ejercicio y el Deporte. 9(1), 73-190.

52. Sousa, D. J., Prudente, J. N., Sequeira, P., López López, J. A. y Hernández-Mendo, A. (2015). Análisis de las situaciones de juego 2vs2 en el campeonato europeo masculino de balonmano 2012: Aplicación de la técnica de coordenadas polares. Cuad. de Psicol. del Deporte, 15(1), 181 


\section{Coordenadas polares para el estudio de los sistemas defensivos en balonmano}

$-194$.

doi.org/10.4321/S1578-

$\underline{84232015000100018 .}$.

53. Vázquez-Diz, J.A., Morillo-Baro, J.P., Reigal, R.E., Morales-Sánchez, V. y Hernández-Mendo, A. (2019a). Mixed Methods in Decision-Making Through Polar Coordinate Technique: Differences by Gender on Beach Handball Specialist. Front. Psychol. 10:1627. https://doi.org/10.3389/fpsyg.2019.01627

54. Vázquez-Diz, J.A., Morillo-Baro, J.P., Reigal, R.E., Morales-Sánchez, V. y Hernández-Mendo, A. (2019b). Contextual Factors and DecisionMaking in the Behavior of Finalization in the Positional Attack in Beach Handball: Differences by Gender Through Polar Coordinates Analysis. Front. Psychol. 10:1386. doi.org/10.3389/fpsyg.2019.01627

55. Volossovitch, A. (2013). Handball. In T. McGarry, P. O’Donoghue and J. Sampaio (Eds.), Routledge Handbook of Sports Performance Analysis (pp. 380-392). New York: Routledge.

56. Vuleta, D., Sporiš, G., Vuleta jr. D., Purgar, B., Herceg, Z. y Milanović, Z. (2012). Influence of attacking efficiency of the outcome of handball matches in the preliminary round of men' s olimpic games 2008. Sport Science 5(2), 7-12. 